\title{
MASSIVE OPEN ONLINE COURSES (MOOCS): PERFIL DOS CURSOS NO CAMPO DA SAÚDE
}

\author{
Liara Saldanha Brites - UFRGS - liarabrites@ yahoo.com.br \\ Cristianne Maria Famer Rocha - UFRGS - cristianne.rocha@ufrgs.br
}

\begin{abstract}
Resumo. Diversas instituições de ensino, impulsionadas pela dinâmica evolução do uso das tecnologias, têm explorado as possibilidades de ensino a distância. Assim despontaram os Massive Open Online Courses (MOOC). Este estudo, de cunho exploratório e abordagem quantitativa e qualitativa, a partir da análise de dados do site MOOC-List.com, objetivou realizar um levantamento das características dos MOOCs relacionados à saúde. Os 376 cursos encontrados para área da saúde representam apenas 8,19\% dentre os 4.593 cursos disponíveis, tendo sido a maioria $(82,18 \%)$ ofertada a partir de 2015 . A duração estimada dos MOOCs, bem como o número de horas dedicado para realizar os cursos, são variados. Inglês é o idioma predominante $(81,35 \%)$. A plataforma Coursera, os países Estados Unidos da América e Reino Unido ofertaram mais cursos. O Brasil ocupa a $6^{a}$ posição e somente doze dos MOOCs encontrados são brasileiros. Os MOOCs na saúde são recentes, poucos são disponibilizados em português e produzidos a partir da realidade brasileira. Porém, conhecer suas características contribui no planejamento e implantação de novos cursos.
\end{abstract}

Palavras-chave: MOOC; educação a distância; educação em saúde.

\section{MASSIVE OPEN ONLINE COURSES (MOOCS): PROFILE OF COURSES IN THE HEALTH FIELD}

Abstract. Several educational institutions, driven by the dynamic evolution of the use of technologies, have explored the possibilities of distance learning. In this context, emerged the Massive Open Online Courses (MOOC). This study has an exploratory design and a quantitative and qualitative approach, and analyzes the MOOC directory, MOOC-List.com, aimed to survey the characteristics of MOOCs about health. The 376 courses found for health represent only $8.19 \%$ from the 4.593 courses available, the majority (82.18\%) offered from 2015. The estimated duration of MOOCs and an estimated dedication of hours per week to take the courses are varied. English is the predominant language (81.35\%). The Coursera platform and the United States of America and the United Kingdom had offered more courses. Brazil occupies the 6th position and only twelve courses are Brazilian. The MOOCs in health are recent, few are available in Portuguese and produced from the Brazilian reality. However, knowing its characteristics contributes to the planning and implementation of new courses.

Keywords: MOOC; education; education, distance; health education. 


\section{Contextualização dos MOOCs}

Ao longo dos anos, teorias de aprendizagem se adequaram, surgiram ou foram modeladas para atualizar a educação, compartilhando espaço em ambientes virtuais, onde pessoas e conhecimentos diversos despertam uma nova forma de se relacionar, ensinar e aprender. Diversas instituições de ensino, impulsionadas pela dinâmica evolução do uso das tecnologias, têm explorado, por exemplo, as possibilidades de ensino a distância. Nesse cenário, propício a novidades na educação mediada por ferramentas virtuais, despontaram os Cursos Online Massivos e Abertos (MOOC, abreviação em inglês de Massive Open Online Courses), objetos deste estudo. Os MOOCs têm origem no Canadá. A sigla foi utilizada pela primeira vez, em 2008, por Dave Cormier, em alusão ao Curso de George Siemens e Stephen Downes, o Connectivism and Connective Knowledge, ministrado online, com acesso aberto as 2.300 pessoas que se registraram, apesar de apenas 25 delas terem pago uma taxa para receberem certificação ao final (Yuan; Powell, 2013).

Os MOOCs são cursos ofertados gratuitamente para qualquer pessoa com acesso à internet, de curta duração (de 3 a 12 semanas, geralmente). Em média, são promovidos e coordenados por renomadas instituições de ensino e disponibilizados em plataformas virtuais de aprendizagem, em inúmeras opções e, em algumas situações, com certificação paga a quem desejar, em distintas áreas do conhecimento (Dal Forno; Knoll, 2013). Apesar das características em comum (por serem cursos abertos, online e massivos), Cisel \& Bruillard (2012) compreendem que não há um consenso sobre a definição de MOOC. O primeiro MOOCs se referia à definição original, baseada no conceito de conectivismo, porém, com o surgimento das plataformas americanas Coursera, Udacity e edX, houve mudanças.

As características dos MOOCs levaram autores a pesquisar e a criar distintas classificações (Lane, 2012; Clarck, 2013), sendo duas as vertentes mais clássicas de MOOCs: os $c M O O C s$ e os $x M O O C s$. Um exemplo de $c M O O C s$ é o primeiro MOOC precitado, baseado no conectivismo. Os $c M O O C s$ apostam na geração de conhecimento por parte dos estudantes, que são estimulados a buscar mais informações na rede e, desta forma, evoluir nos debates e aumentar a interatividade entre os participantes nas plataformas (blogs, wikis, websites, redes sociais, etc.) (Dal Forno; Knoll, 2013; Cisel; Bruillard, 2012). Já os recentes xMOOCs, em geral, são centrados na figura de um professor que define os formatos do curso, onde a prioridade é a transmissão de conteúdos e não a interatividade entre os aprendizes (Dal Forno; Knoll, 2013).

Independente da classificação, os MOOCS abordam conteúdos de distintas áreas de conhecimento, embora não em pé de igualdade. Ao realizar buscas nos sites de grandes plataformas (como Coursera, Udacity, Veduca, edX e MiríadaX), é possível observar que se destacam as Ciências, Tecnologia, Engenharia e Matemática, o que pode ser atribuído à questão histórica dos primeiros MOOCs serem destas áreas e também ao fato de grandes universidades parceiras das essas plataformas serem consagradas nestas áreas. Na saúde, em que atuam as autoras, por exemplo, são observados poucos MOOCs e uma baixa produção científica internacional sobre os assuntos "MOOC" e "saúde" (BRITES \& ROCHA, 2017).

Além dos sites das plataformas, é possível obter informações de milhares de MOOCs por meio do MOOC-List.com. Trata-se de um diretório de MOOCs, em língua inglesa, que divulga cursos disponíveis em 90 provedores e com acesso aberto, embora seja permitida a opção de pagamento para certificação ou exame. Nele, mediante cadastro gratuito, é possível encontrar MOOCs utilizando vários filtros disponíveis em uma ferramenta de busca, como: áreas do conhecimento, novos cursos, cursos com início em breve, fornecedor, universidades, duração, categorias, tempo estimado de estudo semanal, idioma, país e lista de palavras-chave. É importante ressaltar que o site não distingue os cursos em $x M O O C$ ou $c M O O C$ e não se propõe aqui a realizar esta categorização, embora seja possível afirmar que conhecidas plataformas de $x M O O C$, como Coursera, Udacity, edX e MiríadaX estão listadas no diretório. 
Desta forma, este estudo objetiva realizar um levantamento das características dos cursos relacionados à saúde e oferecidos, até maio de 2016, pelas maiores plataformas de MOOCs, a fim de embasar a criação de novos cursos para profissionais e população em geral interessados na área da saúde.

\section{Caminhos metodológicos}

Trata-se de estudo de cunho exploratório e de abordagem quantitativa e qualitativa. A partir de busca no site do MOOC-List.com, um banco de dados foi criado para análise dos cursos. Primeiro, dentre as 32 categorias (opções de busca disponíveis no site por temática abordada), selecionou-se no site apenas aquela denominada "saúde e sociedade". Assim, foram localizados 376 cursos, apresentados brevemente no site. Cada nome de curso contém, no MOOC-List.com, um hiperlink com acesso a uma nova página virtual com detalhamento (nome, provedor, instituição de ensino, instrutores, categoria, dia de início do curso, duração, prérequisitos, tipo de trabalho final, idioma, país e recursos didáticos utilizados). Estes elementos, coletados individualmente, foram agregados em um banco de dados. Foram incluídos todos os cursos disponíveis no diretório (independente da sua data de término ou começo) até a data de 22 de maio de 2016 (data da última coleta) e nenhum curso, dentre os 376 selecionados, foi excluído da análise. Uma vez conformando o banco de dados de MOOCs, foi realizado um levantamento das ocorrências, para posterior análise e categorização.

\section{Os MOOCs no campo da saúde}

A área da saúde tem pouca exibição no universo de MOOCs quando comparada a outras áreas de conhecimento, como Ciências da Computação e Engenharias. Os 376 cursos encontrados na categoria "saúde e sociedade" representam apenas 8,19\% dentre os 4.593 cursos disponíveis no diretório.

Nem todos os MOOCs têm sua data inicial disponibilizada no MOOC-List.com, sendo que 45 apresentam informações como "anunciado" (não foram lançados, mas já estão anunciados no site), "arquivado", "não disponível" ou "individualizado" (este possui características auto instrucionais, onde o interessado realiza o curso conforme seu desejo e sua disponibilidade).

Sobre os 331 cursos que apresentam data, é possível observar a criação recente e o crescimento de MOOCs na saúde ao longo dos anos. Apesar dos MOOCs terem sido criados em 2008 e o ano de 2012 ser considerado "o ano dos MOOCs" (Pappano, 2012) no mundo, devido ao rápido crescimento e à visibilidade alcançada pelos cursos naquele ano, tanto pelo número de alunos inscritos quanto pelo número de renomadas instituições de ensino que passaram a ofertar cursos por meio das plataformas provedoras, não há registro de curso no MOOC-List.com, no campo da saúde, naquele ano. Os primeiros são datados de 2013, quando 8 cursos foram disponibilizados, e observa-se um aumento até 2015 ( $n=143)$. Em 2016, de janeiro a maio, é possível observar 129 cursos. Somados aos cursos de 2015, representam 82,18\% (n=272) da oferta de MOOCs na área da saúde, o que demonstra um aumento de produção para a área.

Os 376 cursos da saúde pertencem a $25(27,78 \%)$ das 90 plataformas listadas no $M O O C$ List.com. A americana Coursera tem ofertado mais cursos na área da saúde $(37,77 \%, \mathrm{n}=142)$, seguida da britânica FutureLearn $(15,69 \%, \mathrm{n}=59)$, das americanas $e d X(14.36 \%, \mathrm{n}=54)$ e Canvas $(7.18 \%, \mathrm{n}=27)$, da ibero-americana MiríadaX $(5.32 \%, \mathrm{n}=20)$ e da francesa France Université Numérique - FUN $(4.79 \%, \mathrm{n}=18)$.

As plataformas provêm MOOCs com conteúdos elaborados pelas instituições de ensino nas quais elas têm firmado parcerias. Assim, as três plataformas com mais MOOCs na saúde possuem parcerias com instituições que possuem experiência na saúde. Em estudo sobre a visão de especialistas americanos e brasileiros sobre os MOOCs, Jacoski (2015) apontou que a 
Coursera detém mais instituições, mais cursos e vem crescendo significativamente, o que também pode explicar sua liderança em cursos para a saúde. Segundo o site da Coursera, esta possui atualmente parceria com 150 instituições de 29 países (Coursera, 2017), dentre elas algumas com renome de produções técnico-científicas no campo da saúde, como: Johns Hopkins Bloomberg School of Public Health, Stanford University, University of Copenhagen, Yale University, University of Michigan e The University of Manchester. Entre as parceiras da Futurelearn estão a University of Birmingham, London School Of Hygiene \& Tropical Medicine, University Of Leeds e Taipei Medical University. Já a edX conta com as renomadas Harvard University, University of Toronto, Boston University, dentre outras.

As informações relacionadas ao país e idioma de origem de cada MOOC também estão intimamente ligadas a essa parceria entre instituições e plataformas. Em geral, uma universidade brasileira, por exemplo, produzirá o MOOC pelo menos na língua mãe (português, podendo haver tradução para outras línguas) e este será disponibilizado na plataforma parceira desta instituição.

Observa-se que 28 países produzem MOOCs para a saúde, sendo os Estados Unidos da América o líder (43.62\%, $\mathrm{n}=164)$, seguido do Reino Unido $(18.35 \%, \mathrm{n}=69)$, da França (5.85\%, $\mathrm{n}=22)$, da Espanha $(5.59 \%, \mathrm{n}=21)$ e da Austrália $(5.05 \%, \mathrm{n}=19)$. O Brasil ocupa a $6^{\mathrm{a}}$ posição, com3.19\% $(\mathrm{n}=12)$ dos cursos.

Os dados acima são compatíveis com os idiomas encontrados. Ao todo, encontram-se MOOCs em até 10 línguas, sendo a maioria $(97,1 \%, n=367)$ ofertada em apenas uma língua, enquanto os demais $(2,39 \%, n=9)$ são apresentados em inglês e mais uma ou duas línguas. $\mathrm{O}$ inglês é predominante, está presente em $81,35 \%(\mathrm{n}=314)$ dos cursos, seguido do espanhol $(6,99 \%, n=27)$, francês $(5,18 \%, n=20)$ e português $(3.37 \%, n=13)$. Os resultados convergem com o estudo de Finardi \& Tyler (2015) que encontraram, dentre os 2.800 MOOCs analisados, 2.326 deles $(83 \%)$ em inglês.

Em estudo sobre o papel e a relação entre globalização e línguas estrangeiras no processo de internacionalização do ensino superior, Finardi et al (2016) discutem sobre a liderança das universidades que oferecem MOOC em inglês também ser encontrada no ranking das melhores universidades do mundo.

Ao analisar o World Reputation Rankings 2016 (World Reputation Rankings, 2016), é possível observar que oito instituições americanas estão entre as dez melhores universidades do mundo (todas anglófonas), possuem parcerias com a Coursera e a $e d X$, que ofertam curso na área da saúde, e produzem MOOC, predominantemente, em inglês: 
Quadro 1.Ranking das melhores universidades do mundo, país e plataformas parceiras

\begin{tabular}{|c|c|c|c|c|}
\hline INSTITUIÇÃO & PAÍS & $\begin{array}{l}\text { IDIOMA } \\
\text { PRINCIPAL }\end{array}$ & $\begin{array}{l}\text { POSIÇÃO } \\
\text { EM } 2016\end{array}$ & $\begin{array}{l}\text { PLATAFORMA } \\
\text { PROVEDORA DE } \\
\text { MOOC }\end{array}$ \\
\hline Universidade de Harvard & $\begin{array}{l}\text { Estados } \\
\text { Unidos da } \\
\text { América } \\
\end{array}$ & Inglês & 1 & edX \\
\hline Instituto de Tecnologia de Massachussets & $\begin{array}{l}\text { Estados } \\
\text { Unidos da } \\
\text { América } \\
\end{array}$ & Inglês & 2 & edX \\
\hline Universidade de Stanford & $\begin{array}{l}\text { Estados } \\
\text { Unidos da } \\
\text { América } \\
\end{array}$ & Inglês & 3 & Coursera \\
\hline Universidade de Cambridge & Reino Unido & Inglês & 4 & $\begin{array}{l}\text { Ofertam cursos } \\
\text { online, mas não são } \\
\text { considerados MOOC }\end{array}$ \\
\hline Universidade de Oxford & Reino Unido & Inglês & 5 & $\begin{array}{l}\text { Ofertam cursos } \\
\text { online, mas não são } \\
\text { considerados MOOC }\end{array}$ \\
\hline Universidade da Califórnia, Berkley & $\begin{array}{l}\text { Estados } \\
\text { Unidos da } \\
\text { América } \\
\end{array}$ & Inglês & 6 & edX \\
\hline Universidade de Princeton & $\begin{array}{l}\text { Estados } \\
\text { Unidos da } \\
\text { América }\end{array}$ & Inglês & 7 & Coursera \\
\hline Universidade de Yale & $\begin{array}{l}\text { Estados } \\
\text { Unidos da } \\
\text { América } \\
\end{array}$ & Inglês & 8 & Coursera \\
\hline Universidade de Columbia & $\begin{array}{l}\text { Estados } \\
\text { Unidos da } \\
\text { América } \\
\end{array}$ & Inglês & 9 & edX e Coursera \\
\hline Instituto de Tecnologia da Califórnia & $\begin{array}{l}\text { Estados } \\
\text { Unidos da } \\
\text { América } \\
\end{array}$ & Inglês & 10 & Coursera \\
\hline
\end{tabular}

Fonte: sistematização das autoras (2016)

São necessários conhecimento de inglês e letramento digital para acessar informações online e participar ativamente no mundo globalizado e a língua inglesa ainda predomina nos conteúdos online (Finardi; Tyler, 2015), mas Altbach (2014) alerta sobre um possível controle de conhecimento. Segundo o autor, os conteúdos dos MOOCs são baseados em experiências acadêmicas e ideias pedagógicas norte-americanas e, mesmo quando se tenta diversificar com traduções para outros idiomas, tende a predominar o contexto americano ou europeu, já que estes dominam a literatura científica e possuem professores de renome que dão aulas e pesquisam em universidades de prestígio. Nesse sentido, Boal \& Stallivieri (2015) também apontam que os MOOCs devem considerar uma internacionalização curricular e atentarem para diferentes pontos de vista, contemplando distintas visões de mundo.

Ainda sobre os resultados de MOOCs deste estudo, a duração estimada de cada MOOCs encontrado é bem variada, sendo que no MOOC-List.com não há informação sobre 3 cursos e $10,90 \%(n=41)$ daqueles encontrados possuem características auto instrucionais, portanto, não apresentam uma sugestão de duração. Nos demais 332 cursos, estima-se uma duração de 1 a 16 semanas, sendo que a maioria $(81,63 \%, n=271)$ se concentra entre 4 e 8 semanas de duração.

Também há uma estimativa da dedicação de horas semanais que o aluno precisa ter para realizar o curso. No caso dos cursos da saúde, é possível observar, entre os 351 MOOCs $(n=351)$ que apresentam essa informação, uma diversidade de resultados, variando de 1 a 18 horas de estudo semanal. 
Mattar (2013) alerta que, em função da diversidade de cursos, plataformas, métodos pedagógicos, instituições e modelos de negócio que já caracterizam o universo dos MOOCs, algumas definições de MOOC como sendo sempre um curso, aberto, massivo e online, não são extensivas a todas as experiências existentes. Em alguns casos, há cobrança de taxas para certificação e a tendência é de que alguns MOOCs venham a ser pagos, como em universidades americanas, nas quais já há um movimento para que os MOOCs sejam reconhecidos e validados como disciplinas de graduação ou pós-graduação. Muitos cursos divulgados como MOOCs também exigem inscrição e o estudante participa em uma plataforma fechada. Além disso, nem todos os materiais têm código aberto ou são Recursos de Educação Aberta (REA), já que muitos estão protegidos por algum tipo de propriedade intelectual.

A maioria dos cursos para a saúde não exige exame ou produção de projeto ao final $(75,27 \%, n=283)$. Dentre os $93(24,73 \%)$ que não exigem, 15 são pagos. Com relação à certificação de conclusão do curso, não há esta informação em 34 deles, mas 90,35\% (n=309) dos informados oferecem certificados e 9,65\% $(n=33)$ não.

Quanto às exigências iniciais, são apontadas informações sobre recomendações prévias à inscrição em cada curso, mas observa-se que não são pré-requisitos, pois não impedem o acesso. Esta característica está relacionada ao fato do MOOC-List.com incluir apenas cursos abertos.

Em estudo sobre o "admirável mundo novo" (Spring, 2016, p. 84) dos MOOCs e o papel do bibliotecário na saúde, a autora discorre sobre o imenso potencial dos MOOCs no fornecimento de educação em saúde no terceiro mundo e em países em desenvolvimento, que podem trazer temas que vão desde a adesão de medicamentos, à influência da genética para o desenvolvimento de câncer e à disponibilização de guias para iniciantes sobre saúde cardiovascular. Já Hoy (2014) aponta a importância dos MOOCs na educação continuada dos profissionais de saúde e na educação em saúde com os pacientes, nos quais esses poderiam se beneficiar em comunidades de apoio e em ter acesso a conhecimentos atualizados.

\section{Produção brasileira de MOOCs na área da saúde}

Em um dos poucos trabalhos brasileiros sobre MOOCs, Marques (2015) refere que estes são recentes no Brasil, tendo início em 2012. Já a disponibilização de cursos de universidades brasileiras nos portais americanos teve início somente dois anos depois, em setembro de 2014, em um acordo entre a Universidade Estadual de Campinas (UNICAMP) e a Universidade de São Paulo (USP) com a Coursera, no qual as instituições se comprometeram em produzir e disponibilizar MOOC em diversas áreas do conhecimento, em português, porém com tradução em outros idiomas (Souza; Cypriano, 2016).

Os sites das plataformas informam a existência de parcerias com instituições e organizações brasileiras. A Coursera, além da USP e UNICAMP, com a Fundação Lemann, o Instituto de Ensino Superior e Pesquisa (Insper), o Instituto Tecnológico de Aeronáutica (ITA) e a Fundação Instituto de Administração. A MiríadaX, com a Pontifícia Universidade Católica do Rio Grande do Sul (PUCRS), Fundação Universidade Regional de Blumenau (FURB), Universidade Anhembi Morumbi, Universidade do Vale do Rio dos Sinos (UNISINOS), USP e Universidade Federal do Rio Grande do Sul (UFRGS).. A FutureLearn e a edX ainda não possuem vínculo com instituições brasileiras.

A Veduca, além da parceria, também é brasileira. Iniciou as atividades em $2012 \mathrm{com}$ um acervo aulas das melhores universidades do mundo (como o Massachusetts Institute of Technology- MIT, Harvard, Yale, Stanford e USP), com legendas de vídeo aulas em português e, em 2013, lançou os primeiros MOOCs da América Latina, com certificação. Atualmente, além da USP, a Veduca trabalha com a Universidade de Brasília (UnB), a Universidade Estadual de São Paulo (UNESP), a Fundação Lemann e Instituto Península, a Universidade Federal de Santa Catarina (UFSC), a UNISINOS e a UNICAMP (Veduca, 2016). 
Apesar da contribuição dessas instituições brasileiras para os MOOCs, na área da saúde ainda há pouca representatividade. O quadro abaixo (quadro 4) representa os 12 cursos encontrados ao restringirmos ao filtro "saúde e sociedade" ao país "Brasil", ou seja, somente 3,19\% dos 376 MOOCs são brasileiros. Pode-se observar que 10 estão alocados na plataforma TELELAB e são produzidos pela UFSC; 1 na Coursera, produzido pela USP; e 1 da MiríadaX, produzido pela PUCRS.

Com exceção dos MOOCs da Coursera e MiríadaX, os cursos da TELELAB têm foco em patologias (identificação, monitoramento, tratamento e cuidado), têm características auto instrucionais de aprendizado, estimativa de dedicação semanal de 1 hora, possuem exame e fornecem certificado de conclusão. Essas características podem estar relacionadas às características da instituição que a produz (UFSC) e à plataforma TELELAB, pertencente ao Ministério da Saúde (MS) e que disponibiliza cursos visando os profissionais de saúde.

Quadro 4. Características dos MOOCs brasileiros

\begin{tabular}{|c|c|c|c|c|c|c|c|}
\hline Nome do MOOC & Ano & $\begin{array}{l}\text { Platafor } \\
\text { ma }\end{array}$ & $\begin{array}{l}\text { Institui } \\
\text { ção }\end{array}$ & Duração & $\begin{array}{l}\text { Carga } \\
\text { horária } \\
\text { estimada }\end{array}$ & Exame & $\begin{array}{l}\text { Certifi } \\
\text { cado }\end{array}$ \\
\hline $\begin{array}{l}\text { Big Data em Saúde no } \\
\text { Brasil }\end{array}$ & 2015 & Coursera & USP & 6 semanas & $\begin{array}{l}2-3 \\
\text { horas/semana }\end{array}$ & Sim & Sim \\
\hline $\begin{array}{l}\text { Biossegurança - } \\
\text { Laboratórios de DST, } \\
\text { Aids e Hepatites Virais }\end{array}$ & $\begin{array}{l}\text { Auto } \\
\text { Individu } \\
\text { alizado } \\
\text { (não há } \\
\text { data) }\end{array}$ & TELELAB & UFSC & Individualizado & 15 horas & Sim & Sim \\
\hline $\begin{array}{l}\text { Coleta de sangue - } \\
\text { Diagnóstico e } \\
\text { monitoramento das DST, } \\
\text { Aids e Hepatites Virais }\end{array}$ & $\begin{array}{l}\text { Individu } \\
\text { alizado }\end{array}$ & TELELAB & UFSC & Individualizado & 15 horas & Sim & Sim \\
\hline $\begin{array}{l}\text { Cultura, Isolamento e } \\
\text { identificação da } \\
\text { Neisseriagonorrhoeae }\end{array}$ & $\begin{array}{l}\text { Individu } \\
\text { alizado }\end{array}$ & TELELAB & UFSC & Individualizado & 15 horas & Sim & Sim \\
\hline $\begin{array}{l}\text { Diagnóstico de Hepatites } \\
\text { Virais }\end{array}$ & $\begin{array}{l}\text { Individu } \\
\text { alizado }\end{array}$ & TELELAB & UFSC & Individualizado & 15 horas & Sim & Sim \\
\hline Diagnóstico de HIV & $\begin{array}{l}\text { Individu } \\
\text { alizado }\end{array}$ & TELELAB & UFSC & Individualizado & 15 horas & Sim & Sim \\
\hline Diagnóstico de Sífilis & $\begin{array}{l}\text { Individu } \\
\text { alizado }\end{array}$ & TELELAB & UFSC & Individualizado & 15 horas & Sim & Sim \\
\hline $\begin{array}{l}\text { Doença de Chagas - } \\
\text { Triagem e diagnóstico } \\
\text { sorológico em Unidades } \\
\text { Hemoterápicas e } \\
\text { Laboratórios de Saúde } \\
\text { Pública }\end{array}$ & $\begin{array}{l}\text { Individu } \\
\text { alizado }\end{array}$ & TELELAB & UFSC & Individualizado & 15 horas & Sim & Sim \\
\hline $\begin{array}{l}\text { Doença Falciforme - } \\
\text { Conhecer para Cuidar }\end{array}$ & $\begin{array}{l}\text { Individu } \\
\text { alizado }\end{array}$ & TELELAB & UFSC & Individualizado & 15 horas & Sim & Sim \\
\hline $\begin{array}{l}\text { Equipamentos - Utilização } \\
\text { e monitoramento em } \\
\text { Unidades Hemoterápicas } \\
\text { e Laboratórios de Saúde } \\
\text { Pública }\end{array}$ & $\begin{array}{l}\text { Individu } \\
\text { alizado }\end{array}$ & TELELAB & UFSC & Individualizado & 15 horas & Sim & Sim \\
\hline $\begin{array}{l}\text { Responsabilidade Social e } \\
\text { Sustentabilidade das } \\
\text { Organizações }\end{array}$ & 2015 & Miríada $X$ & PUCRS & 6 semanas & $\begin{array}{l}\text {-4 } \\
\text { horas/semana }\end{array}$ & Não & Sim \\
\hline $\begin{array}{l}\text { Técnicas de Coloração de } \\
\text { Gram }\end{array}$ & $\begin{array}{l}\text { Individu } \\
\text { alizado }\end{array}$ & TELELAB & UFSC & Individualizado & 15 horas & SIm & Sim \\
\hline
\end{tabular}

Fonte: sistematização das autoras (2016) 
Além dessa parceria com o TELELAB, o MS vem apostando em outras iniciativas importantes no movimento de cursos online e abertos para a Saúde Pública/Coletiva (embora não sejam reconhecidos institucionalmente como MOOCs), como aqueles ofertados pela Universidade Aberta do SUS (UNA-SUS), o Ambiente Virtual de Aprendizagem do SUS (AVA-SUS) e a Comunidade de Práticas (CdP). O Ministério da Educação (MEC) também apresenta cursos similares por meio da Universidade Aberta do Brasil (UAB).

\section{Considerações}

Embora este estudo não abranja todos os MOOCs, uma vez que pode haver cursos não listados no MOOC-List.com, nossa intenção foi conhecer as características dos MOOCs já existentes, o que pode contribuir com reflexões e discussões acerca do planejamento e implantação de MOOCs para participantes e instituições interessados em temas da área da saúde.

Ao apreciar as diversas plataformas e cursos, é possível perceber que a pluralidade de formatos e usos dos MOOCs dificulta enquadrá-los em um único conceito. A variedade dos MOOCs quanto à duração, aos temas, à estimativa de dedicação de carga horária, à certificação, à exigência de exames ou à produção de projeto final, também tem relação intrínseca ao perfil da plataforma provedora e da experiência da instituição de ensino nos conteúdos produzidos.

O inglês predomina como idioma dos MOOCs para a saúde, o que se explica em parte pelo investimento das plataformas americanas e universidades americanas, mas também simboliza a manutenção do poder destas instituições no mundo ao divulgarem amplamente suas ideologias e conhecimentos no campo da saúde e com base em suas realidades locais. Nem mesmo o aumento de MOOCs para a saúde e de produção de MOOCs por instituições brasileiras, algumas com expertise na área e firmando acordos com grandes plataformas, têm sido significativo para se encontrar MOOCs em português, produzidos a partir da realidade brasileira, com temas voltados às questões de saúde e diminuindo barreiras linguísticas. Também é preciso reconhecer limitações do estudo, já que o MoocList.com é um catálogo internacional, em língua inglesa, que referencia principalmente instituições oriundas de países de língua inglesa e a tradução para português nas plataformas estrangeiras pode resultar numa sobrecarga no desenvolvimento dos cursos, o que pode refletir em baixa produção de cursos em português.

Os MOOCs com conteúdos relacionados à área da saúde ainda têm pouca representatividade, por hipóteses diversas, como: as instituições parceiras das plataformas terem mais experiências em outras áreas de conhecimento ou haver maior interesse comercial em outras áreas em detrimento da área da saúde. Um dos obstáculos prováveis seja o olhar biomédico para aspectos clínicos e biológicos (que impõe barreiras éticas sobre a responsabilidade ao disponibilizar conhecimento como sobre procedimentos clínicos e diagnósticos, restritos a algumas categorias profissionais, amplamente para a população) em detrimento da perspectiva da Saúde Coletiva/Pública de um trabalho de educação em saúde, potencializando a autonomia dos sujeitos e o acesso à informação em saúde. É importante que haja mais estudos específicos para estas e outras questões que neste trabalho não foi possível investigar.

As ofertas de MOOCs na área de saúde devem considerar, principalmente: a educação permanente e continuada dos trabalhadores; a troca de experiências entre estudantes, profissionais e pacientes de diversos locais do mundo (nos quais, por exemplo, uma mesma patologia ou até uma epidemia pode se manifestar de forma diferente); e o conhecimento como modo de fortalecer a autonomia e melhorar as condições de saúde da população. Um país onde já se faz uso de educação mediada por ferramentas online, com um grande número de profissionais de saúde e milhões de pessoas acompanhadas por um dos maiores sistemas de saúde pública do mundo e que também podem usufruir do conhecimento, parece sinalizar uma 
demanda, ainda que pouco conhecida, e um cenário favorável e pouco explorado ao uso de MOOCs.

\section{Referências}

ALTBACH, Phillip G. Moocs as neocolonialism: who controls knowledge? International higher education, 2014. p. 5-7.

BOAL, Helena Maria Chaves; STALLIVIERI, Luciane. Os MOOCs e o processo de internacionalização das instituições de ensino superior.In: XVColóquio Internacional de Gestão Universitária - CIGU -Desafios da gestão universitária no século XXI, 2015, Mar del Plata, Argentina. Disponível em:

<https://repositorio.ufsc.br/bitstream/handle/123456789/136147/102_00012.pdf?sequence=1\&i sAllowed=y>. Acesso em: 5 set. 2016.

BRITES, Liara Saldanha Brites; ROCHA, Cristianne Maria Famer. Massive Open Courses (Mooc) in health field: brazilian iniciatives. In: NETO, André Pereira. Internet and Health in Brazil: challenges and trends (no prelo). New York: Springer Science Books, 2017.

CISEL, Matthieu; BRUILLARD, Éric. Chronique des MOOC. Sciences et Technologies de l'Information et de la Communication pour l'Éducation et la Formation, v. 19, 2012. Disponível em: <http://sticef.univ-lemans.fr/num/vol2012/13rcisel/sticef_2012_cisel_13r.htm>. Acesso em: 20 nov. 2015.

CLARCK, Donald. MOOCs: taxonomy of 8 types of MOOC. 2013. Disponível em: <http://donaldclarkplanb.blogspot.com.br/2013/04/moocs-taxonomy-of-8-types-ofmooc.html>. Acesso em: 02 fev. 2016.

COURSERA. 2017. Disponível em: <https://pt.coursera.org/>. Acesso em: 15 jul 2017.

DAL FORNO, Josiane Pozzatti; KNOLL, Graziela Frainer. Os MOOCs no mundo: um levantamento de cursos online abertos massivos. Nuances: estudos sobre educação, Presidente Prudente-SP, v. 24, n. 3, p. 178-194, set./dez. 2013. Disponível em: <http://revista.fct.unesp.br/index.php/Nuances/article/view/2705>. Acesso em: 14 nov. 2015.

FINARDI, Kyria Rebeca; SANTOS, Jane; GUIMARÃES, Felipe. A relação entre línguas estrangeiras e o processo de internacionalização: evidências da coordenação de letramento internacional de uma universidade federal. Interfaces Brasil/Canadá, 2016. v. 16, n. 1, p. 233 255. Disponível em:

<https://periodicos.ufpel.edu.br/ojs2/index.php/interfaces/article/view/7514/5622>. Acesso em: $1^{\circ}$ ago. 2016.

FINARDI, Kyria Rebeca; TYLER, Jhamille Roberts. The role of english and technology in the internationalization of education: insights from the analysis of MOOCs.In: 7th international conference on education and new learning technologies. 2015, Barcelona. Edulearn15 Proceedings... Barcelona: IATED, v. 1. p. 11-18. 2015.

HOY, Matthew B. MOOCs 101: an introduction to Massive Open Online Courses. Medical reference services quarterly, 14 jan. 2014. v. 33, n. 1, p. 85-91. Disponível em: 
<http://www.tandfonline.com/doi/abs/10.1080/02763869.2014.866490>. Acesso em: $1^{\circ}$ ago. 2016.

JACOSKI, Cláudio Alcides. Técnicas de aprendizagem e MOOCs: uma visão de especialistas do Brasil e Estados Unidos da América. Mar del Plata, Argentina: INPEAU/UFSC, 2015. Disponível em: <https://repositorio.ufsc.br/handle/123456789/135879?show=full>. Acesso em: 5 set. 2016.

LANE, Lisa M. Three kinds of MOOCs. Lisa's (Online) Teaching Blog, ago. 2012. Disponível em: <http://lisahistory.net/wordpress/2012/08/three-kinds-of-moocs/>. Acesso em: 15 nov. 2015.

MARQUES, Paula Fogaça. Massive Open Online Course (MOOC) : uma análise de experiências pioneiras. 2015. 91 f. Dissetação (Mestrado) - Programa de Pós-graduação em Educação, Faculdade de Educação, Universidade Federal do Rio Grande do Sul, Porto Alegre, 2015. Disponível em: <http://www.lume.ufrgs.br/handle/10183/131916>. Acesso em: $1^{\circ}$ jan. 2016.

MATTAR, João. Aprendizagem em ambientes virtuais: teorias, conectivismo e MOOC. Teccogs, n. 7, p. 20-40, jan./jun. 2013. Disponível em: <http://www.pucsp.br/pos/tidd/teccogs/artigos/2013/edicao_7/2aprendizagem_em_ambientes_virtuais-joao_mattar.pdf >. Acesso em: 18 nov. 2015.

PAPPANO, Laura. The year off the MOOCs. The new york times, [S.1.], 2012. Disponível em: <http://www.nytimes.com/2012/11/04/education/edlife/massive-open-online-courses-aremultiplying-at-a-rapid-pace.html>. Acesso em: 1 set. 2016.

SOUZA, Rodrigo de; CYPRIANO, Elysandra Figueiredo. MOOC: uma alternativa contemporânea para o ensino de astronomia. Ciência \& educação (Bauru), mar. 2016. v. 22, n. 1, p. 65-80. Disponível em: <http://www.scielo.br/scielo.php?script=sci_arttext\&pid=S1516$73132016000100065 \& \operatorname{lng}=$ pt\&nrm=iso\&tlng=en>. Acesso em: 24 set. 2016.

SPRING, Hannah. Online learning: the brave new world of massive open online courses and the role of the health librarian. Health information \& libraries journal, mar. 2016. v. 33, n. 1, p. 84-88. Disponível em: <http://doi.wiley.com/10.1111/hir.12134>. Acesso em: $1^{\text {o }}$ ago. 2016.

VEDUCA. 2016. Disponível em: <http://www.veduca.com.br/>.Acesso em: 30 jul. 2016.

WORLD REPUTATION RANKINGS 2016.The Times Higher Education. 2016. Disponível em: <https://www.timeshighereducation.com/world-university-rankings/2016/reputationranking\#!/page/0/length/25/sort_by/rank_label/sort_order/asc/cols/rank_only>. Acesso em: 30 jul. 2016.

YUAN, Li; POWELL, Stephen. MOOCs and open education: implications for higher education. Bolton, UK: CETIS/University of Bolton, 2013. Disponível em: <http://publications.cetis.ac.uk/2013/667>. Acesso em: 20 nov. 2015. 